Ferner ist

$$
\begin{gathered}
z=\left[a_{2}+i \alpha_{1}\right] \sqrt{2 \delta_{0}\left(1-\frac{w}{\tilde{\omega}}\right)} ;\left(\frac{w}{\tilde{\omega}}<1\right) \\
\chi=\left[U_{1}+i U_{2}\right] \sqrt{2 \delta_{0}\left(1-\frac{w}{\tilde{\omega}}\right)}, \\
\alpha_{1}=\sqrt{\frac{1}{2}\left(\sqrt{P^{2}+Q^{2}}+P\right)}, \quad \alpha_{2}=\sqrt{\frac{1}{2}\left(\sqrt{\left.P^{2}+Q^{2}-P\right)} ;\right.} \\
U_{1}=\alpha_{2} \cos \left(\frac{\tilde{\omega}}{2} t\right)-\alpha_{1} \sin \left(\frac{\tilde{\omega}}{2} t\right), \\
U_{2}=\alpha_{1} \cos \left(\frac{\tilde{\omega}}{2} t\right)+\alpha_{2} \sin \left(\frac{\tilde{\omega}}{2} t\right), \\
P=\frac{\sum F_{k} F_{j} \sin \Delta_{k j}}{\sum F_{k}{ }^{2}+2 \Sigma F_{k} F_{j} \cos \Delta_{k j}}, \\
Q=\frac{\sum F_{k} F_{j} \cos \Delta_{k j}}{\sum F_{k}{ }^{2}+2 \sum F_{k} F_{j} \cos \Delta_{k j}}, \\
\beta=P \cos \tilde{\omega} t+Q \sin \tilde{\omega} t, \quad \Pi^{2}=D_{12} D_{21} .
\end{gathered}
$$

In dem Ausdruck (12) und (12 a) für die Übergangswahrscheinlichkeit ist besonders bemerkenswert, daß die Ordnungszahl $n$ der Zylinderfunktionen $J_{n}$ und $N_{n}$ mit der Schwankungsfrequenz $\tilde{\omega}$ der Phasenkonfiguration und mit den charakteristischen physikalischen Größen des atomaren Systems sowie des Strahlungsfeldes durch die Relation

$$
n=\sqrt{\frac{4 \Pi^{2} R^{2}}{\hbar^{2} \tilde{\omega}^{2}}+\frac{w^{2}}{\tilde{\omega}^{2}}}
$$

verknüpft ist. In physikalischer Hinsicht hat die Ordnungszahl $n$ die Bedeutung eines Polytropenindex des Problems. Es ergibt sich somit der grundsätzliche Sachverhalt, $d a ß$ bei Berücksichtigung von Phasenschwankungen das Strahlungsproblem des Masers sehr verschieden gestaltete Lösungen annehmen kann. Es läßt sich zeigen, daß der Ausdruck (12) und (12 a) infolge systematischer Entwicklung nach kleinen Amplituden $\delta_{0}$ der Phasenschwankungen im Grenzfall $\delta_{0} \rightarrow 0$ in die bekannte analytische Form der Übergangswahrscheinlichkeit übergeht.

Es ergibt sich, daß bei einer zeitlich unveränderlichen Phasenverteilung die Übergangswahrscheinlichkeit für
Emissions- bzw. Absorptionsprozesse außer von den Parametern des atomaren Systems und von den Amplituden der Schwingungskomponenten insbesondere von der Phasenkonfiguration abhängt und durch diese unter Umständen wesentlich modifiziert werden kann. Daher ist die sich aus der Übergangswahrscheinlichkeit ergebende momentane Quantenleistung und das unter Berücksichtigung von Relaxationseffekten berechnete Leistungsspektrum sowie dessen Bandbreite eine Funktion der Phasenverteilung.

Unter der Voraussetzung synchroner Phasenschwankungen resultieren aus der Ubergangswahrscheinlichkeit (12) entsprechende Modifikationen sowohl für die momentane Quantenleistung als auch für Leistungsspektrum, Bandbreite und Verstärkung.

Die Phasenschwingungen repräsentieren neben dem atomaren System und dem Strahlungsfeld ein drittes Energiereservoir. Der Energietransport zwischen Feld und Materie kann je nach der Phasenkonfiguration durch Mitbeteiligung dieses Energiespeichers hinsichtlich der Richtung des Energieflusses als auch in bezug auf die maximale Höhe und Breite des Leistungsspektrums beeinflußt werden. Die synchronen Phasenschwankungen repräsentieren einen Steuerungsmechanismus der induzierten Emission und Absorption, der sich vielgestaltig auf das Leistungsspektrum und die Verstärkung auswirken kann. Man unterscheidet gemäß Formel (12 b) zweckmäßig Strahlungsvorgänge mit einem für alle Frequenzen $v$ konstanten Polytropenindex $n$ und solche mit konstanter Frequenz $\tilde{\omega}$ der Phasenschwankungen.

Kompliziertere Verhältnisse ergeben sich, wenn man die Voraussetzungen fester Phasendifferenzen und synchroner Schwankungen fallen läßt und annimmt, daß auch die Phasendifferenzen $\Delta_{k j}$ periodische Schwankungen ausführen. - Eine ausführliche Mitteilung dieser Untersuchungen zum Problemkreis des Masers in dieser Zeitschrift ist für einen späteren Zeitpunkt vorgesehen.

Herrn Prof. Dr. G. Jung und Herrn Dr. H. Rüchardt möchte ich für wertvolle Diskussionen, Herrn Studienrat W. MaIer für freundliche Durchführung einer Kontrolle der Rechnungen und den Herren Dr. K. Siebertz, Dr. P. Henninger, Prof. Dr. J. Dosse und Prof. Dr. J. Labus für das dieser Arbeit entgegengebrachte große Interesse vielmals danken.

\section{Zur Sichtbarmachung von Versetzungen für die elektronenmikroskopische Abbildung *}

Von H. Bethge und V. Schmidt

Institut für experimentelle Physik der Universität Halle

(Z. Naturforschg. 14 a, 307-309 [1959]; eingegangen am 9. Februar 1959)

Für verschiedene Stoffe ist es gelungen, an Spaltflächen oder geeignet vorbehandelten Oberflächen durch Anwendung ausgesuchter chemischer Ätzverfahren zur Oberfläche durchstoßende Versetzungen durch die entstehenden Ätzgrübchen sichtbar zu machen. Aus den zahlreichen, zumeist mit dem Lichtmikroskop durchge- führten Untersuchungen seien hier nur die Ergebnisse von Vogel und Mitarbeitern ${ }^{1}$ erwähnt, die am Germanium die in einer Feinkorngrenze angeordneten Versetzungen erstmals eindeutig nachweisen konnten. Werden die angeätzten Oberflächen über geeignete Abdruckverfahren mit dem Elektronenmikroskop beobachtet, so zeigt sich, daß das erhöhte Auflösungsvermögen nichts grundsätzlich Neues - über das vom Lichtmikroskop

* Die hier mitgeteilte Methode wurde erstmals auf dem IV. Internationalen Kongreß für Elektronenmikroskopie in Berlin (Sept. 1958) im Rahmen eines zusammenfassenden Berichtes bekanntgemacht.

1 F. L. Vogel, W. G. Prann, H. E. Corey u. E. E. Thomas, Phys. Rev. 90, 489 [1953]. 
her bekannte hinausgehend - aufzuzeigen vermag. Für den Fall der von uns zahlreich untersuchten Spaltflächen vom Steinsalz, die mit einem Reagens aus Eisessig und $2 \%$ konz. $\mathrm{H}_{2} \mathrm{SO}_{4}$ geätzt wurden, hat dies seine Ursache darin, daß die Kanten der Ätzgrübchen durch den Ätzvorgang sehr verrundet werden und störende Rückstände elektronenmikroskopischer Größenordnung nicht zu vermeiden sind. Für die Ätzung an LiF haben Gilman, Johnston und SEARs ${ }^{2}$ sorgfältig die Bedingungen für die Anätzung von Versetzungen untersucht. In einer nachfolgenden Arbeit ${ }^{3}$ dieser Gruppe sind auch elektronenmikroskopische Aufnahmen der Ätzgrübchen an LiF gezeigt, in denen die vorstehend beschriebenen Mängel sehr viel weniger in Erscheinung treten als bei der Ätzung an $\mathrm{NaCl}$; aber auch hier bleibt der Einwand bestehen, daß durch den Ätzangriff natürlich auch andere Oberflächenstrukturen angegriffen, zumeist eingeebnet und damit der Abbildung entzogen werden. Es ist somit nicht möglich, die besonders interessierenden $\mathrm{Zu}$ sammenhänge zwischen Oberflächenstrukturen elektronenmikroskopischer Größenordnung - z. B. die von uns früher beschriebenen ${ }^{4}$ "Spaltfiguren“ - und Versetzungen zu untersuchen.

Die hier mitzuteilende Möglichkeit, zur Kristalloberfläche durchstoßende Versetzungen zur Abbildung zu bringen, geht von der Überlegung aus, daß nicht nur der Kristallabbau durch Ätzung bevorzugt an Störungen erfolgen sollte, sondern ebenso ein Kristallwachstum. Hierzu wurde im Hochvakuum auf Steinsalzspaltflächen $\mathrm{NaCl}$ aufgedampft. Durch Erwärmung des Kristalles ist hierbei für eine hinreichende Oberflächenbeweglichkeit der auftreffenden Moleküle zu sorgen. Die Aufdampfgeschwindigkeit ist so zu wählen, daß diese wesentlich größer ist als für ein ideales Kristallwachstum zulässig. Diese Forderung soll ausdrücken, daß z. B. beim Vorliegen von atomaren Spaltstufen diese nicht - gemäß der Theorie von Kossel und STranski ${ }^{5}$ oder nach den Vorstellungen von Burton, Cabrera und Frank 6 über das Wachstum auf realen Oberflächen - über die Kristalloberfläche hin weiterwachsen sollen, sondern die Spaltstufen oder Störungen atomarer Größenordnung als Orte bevorzugter Keimbildung wirken.

Die den Bedingungen angepaßte Temperatur des Kristalles wurde im Experiment zu etwa $300^{\circ} \mathrm{C}$ ermittelt. Diese Temperatur lag einige $10^{\circ} \mathrm{C}$ unter derjenigen, bei der im Hochvakuum (nach einer Zeit von 10 min) die erste Ausbildung von Abdampfstrukturen, z. B. eine sehr feine Lamellierung an gröberen Spaltstufen, elektronenmikroskopisch zu beobachten ist. Die Aufdampfgeschwindigkeit lag bei $100-300 \AA \mathrm{sec}^{-1}$ kondensierter NaCl-Schicht. Durch einfache Wägung ergaben sich - unter der freilich nicht zutreffenden Annahme einer homogenen Bedeckung - Dicken der Aufdampfschicht von $10^{3}-10^{4} \AA$. Unter diesen Bedingun-

2 J. J. Gilman, W. G. Johnston u. G. W. Sears, J. Appl. Phys. 29, 747 [1958].

3 J. J. Gilman u. W. G. Johnston, J. Appl. Phys. 29, 877 [1958].

${ }^{4}$ H. Betrige, Fortschr. Min. 35, 156 [1957].

5 W. Kossel, Leipziger Vorträge 1928, S. Hirzel, Leipzig 1923. - I. N. Stranski, Z. Phys. Chem. A 136, 259 [1928]. gen sind die nachstehend mitgeteilten Ergebnisse erzielt worden. Zur experimentellen Durchführung sei bemerkt, daß die Aufdampfung aus Mo-Schiffchen bei einem Vakuum von besser als $10^{-4}$ Torr erfolgte. Der Kristall befand sich in einem Abstand von 8,5 cm zur Aufdampfquelle in einem in Aufdampfrichtung offenen Röhrenofen. Unmittelbar nach der NaCl-Bedampfung und nachfolgender Abkühlung des Kristalles wurde, ohne die Apparatur zu belüften, durch Kohlebedampfung nach Bradeey ${ }^{7}$ der Abdruckfilm aufgebracht. Nach dem Weglösen des Kristalles und der üblichen Präparation auf Netz-Objektträger wurden die Präparate zur Kontraststeigerung mit $\mathrm{ThF}_{4}$ schräg bedampft. Untersucht wurde aus der Schmelze gezogenes Kristallmaterial; die Spaltung erfolgte durch von Hand ausgeführte Schlagspaltung.

In Abb. $1^{*}$ ist eine durch diese Methode zur Abbildung gebrachte Feinkorngrenze wiedergegeben. Es handelt sich um eine aus Schraubenversetzungen aufgebaute „twist-boundary“. Die durchstoßenden Versetzungen sind durch im Mittel $0,2 \mu$ große, gegenüber der Umgebung deutlich bevorzugt aufgewachsene flache Würfel markiert. Besonders bemerkenswert sind die von den einzelnen Versetzungen in Spaltrichtung davonlaufenden Stufen, die sich zumeist zu gröberen Stufen vereinigen. Diese gröberen Stufen sind die aus lichtmikroskopischer Beobachtung bekannten „river-pattern“. Die von den einzelnen Versetzungen ausgehenden Stufen sollten (auf dem unbedampften Kristall) in der Höhe von atomarer Größenordnung sein. Eine durch die bevorzugte Keimbildung längs solcher Stufen bewirkte linienhafte Anordnung der aufwachsenden Kriställchen, die sich deutlich von der regellos verteilten Anordnung auf den nicht gestörten Bereichen unterscheidet, vermittelt die Abbildung und den Verlauf der Stufen.

Ein Gebiet höherer Versetzungsdichte ist in Abb. 2 aufgenommen. Die Annahme, daß es sich hier um Durchstoßpunkte von Versetzungen handelt, scheint aus der Diskussion der zahlreich vorliegenden ähnlichen Aufnahmen und durch Vergleich der durch Ätzung ermittelten Flächendichte gerechtfertigt. Während in Abb. 1 aus der Struktur des ,river-pattern“ auf vorliegende Versetzungen mit Schraubencharakter geschlossen werden kann, ist eine Entscheidung darüber, ob in Abb. 2 Schrauben- oder Stufenversetzungen vorliegen, nicht ohne weiteres $\mathrm{zu}$ treffen. Zwar ist nach einer Theorie von $\mathrm{F}_{\text {RANK }}{ }^{8}$ an durchstoßenden Schraubenversetzungen ein - wie auch die Abbildung zeigt - pyramidenförmiges Wachstum zu erwarten, jedoch soll hieraus noch nicht gefolgert werden, daß in Abb. 2 nur Schraubenversetzungen vorliegen. Eine mögliche Entscheidung sollte durch Untersuchungen zur Feinstruktur der Wachstumspyramiden zu gewinnen sein, die unter Anwendung kürzlich bekannt gewordener Methoden ${ }^{9}$ zur Erzielung

6 W. K. Burton, N. Cabrera u. F. C. Frank, Phil. Trans. Roy. Soc., Lond. A 243, 299 [1951].

7 D. E. Bradley, Brit. J. Appl. Phys. 5, 65 [1954].

8 F. C. Frank, Adv. Phys. 1, 91 [1952].

9 D. E. Bradeey, Vortrag auf dem IV. Internat. Kongreß für Elektronenmikroskopie, Berlin 1958.

* Abb. 1 bis 3 auf Tafel S. 308 a. 
H. Bethge und V. Sснмidt, Zur Sichtbarmachung von Versetzungen für die elektronenmikroskopische Abbildung (S. 307).
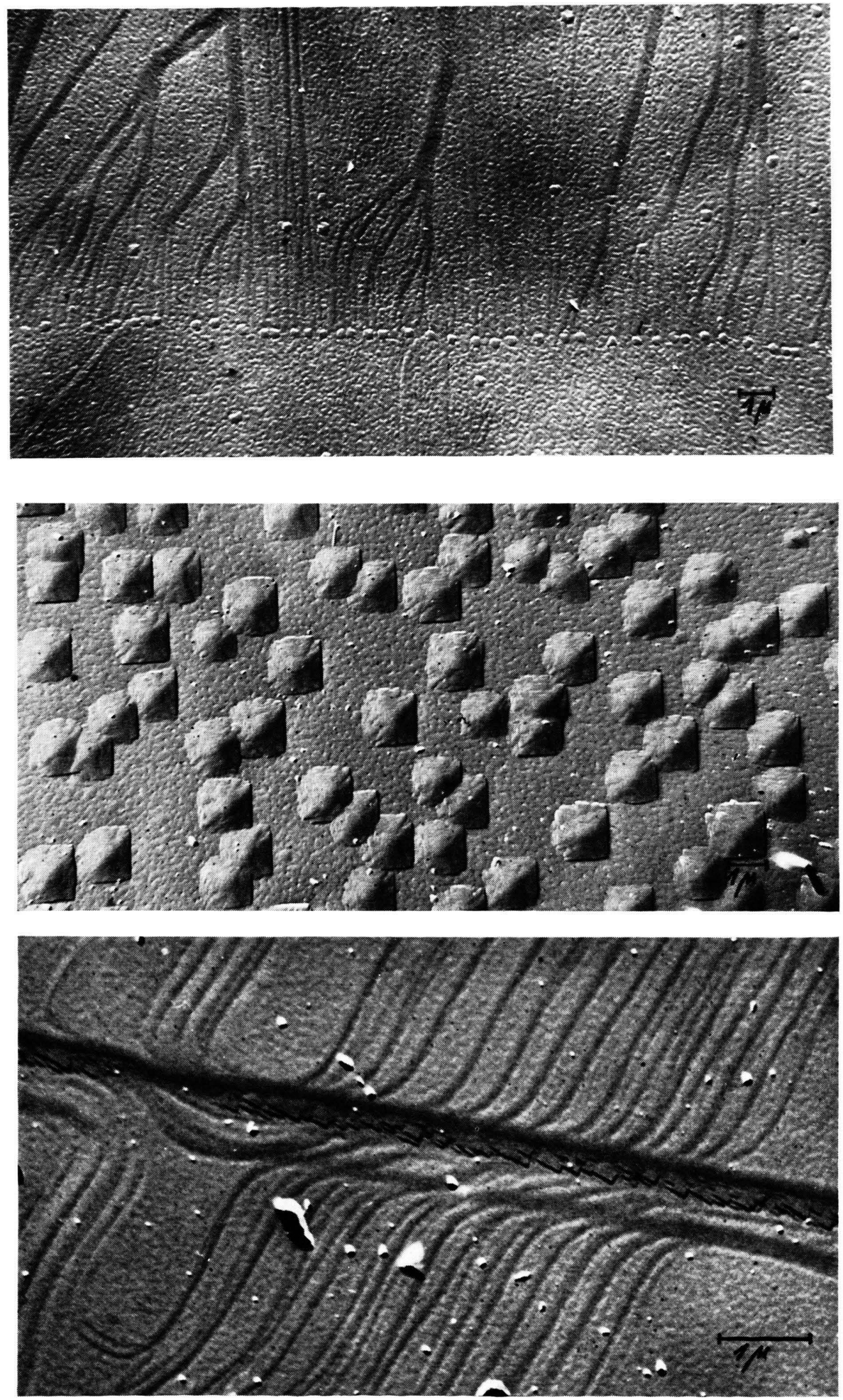

Abb. 1. Feinkorngrenze (twist-boundary) auf einer Steinsalz-Spaltfläche (4800-fach).

Abb. 2. Bereich höherer Versetzungsdichte auf einer Steinsalz-Spaltfläche $(5600-f a c h)$.

Abb. 3. Spaltstrukturen auf einer Steinsalz Spaltfläche, die auf eine Beeinflussung durch beim Spaltvorgang betätigte Versetzungen schließen lassen (13 000-fach). 
W. D. Dachselt und G. Pfefferkorn, Zum Wachstum von Kupferoxydnadeln (S.309).

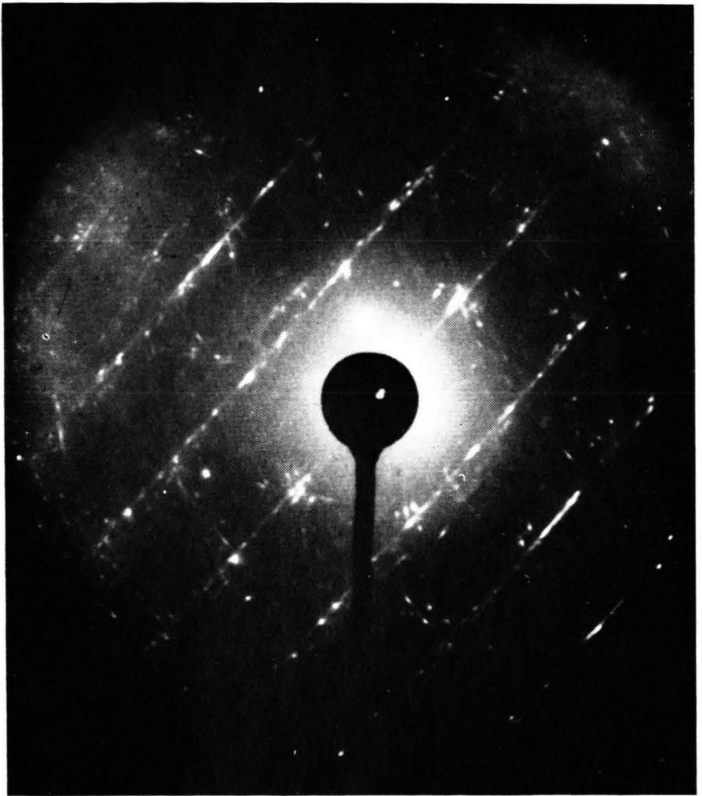

Abb. 1. Elektronenbeugungstexturdiagramm paralleler $\mathrm{CuO}$ Nadeln (oxydiert $15 \mathrm{Stdn}$. bei $200{ }^{\circ} \mathrm{C}$ im Sauerstoffstrom unter HCl-Zusatz).

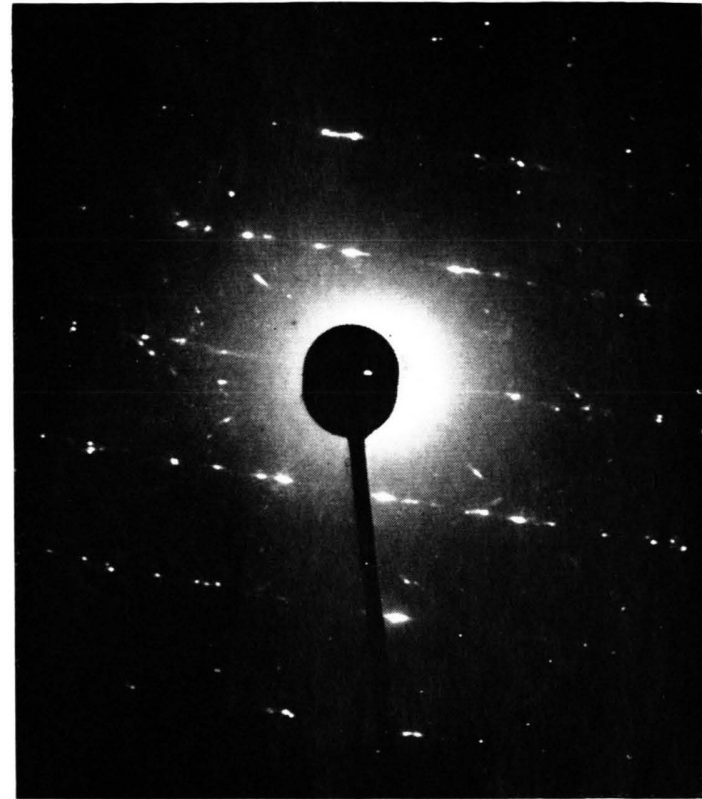

Abb. 2. Elektronenbeugungsdiagramm vorwiegend von einer CuO-Nadel (oxvdiert 9 Stdn. bei $240{ }^{\circ} \mathrm{C}$ im Sauerstoffstrom unter $\mathrm{HCl}-\mathrm{Zusatz}$.

H. Barth u. R. Hosemaxx, Anwendung der Parallelstrahlmethode im Durchstrahlungsfall zur Prüfung des Kristallinneren mit Röstgen-Strahlen (Band $\mathbf{1 3}$ a. S. 792 ; die Abb. 2 bis 4 werden zur Verdeutlichung hier noch einmal auf einer Tafel wiederholt).

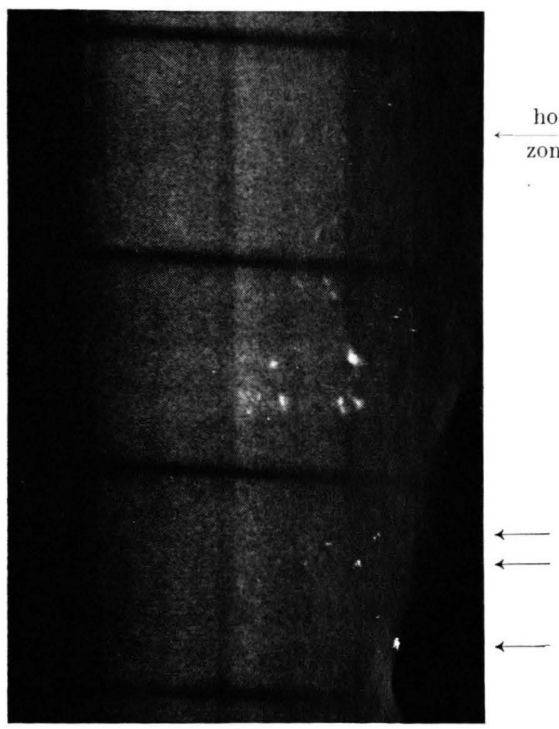

Abb. 2.

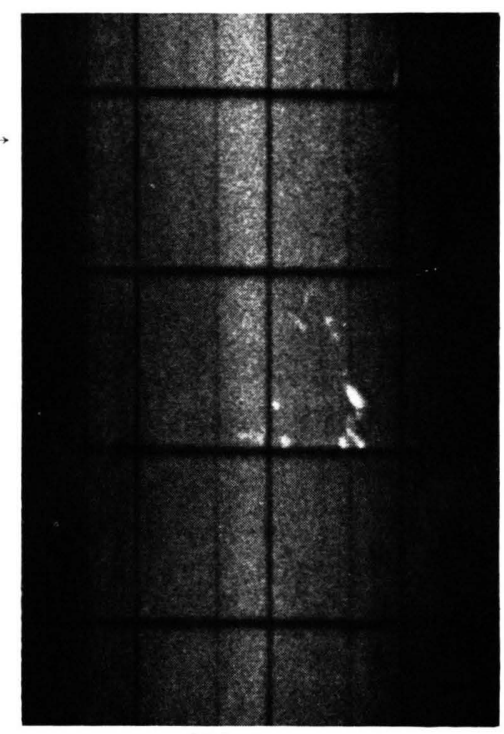

Abb. 3.

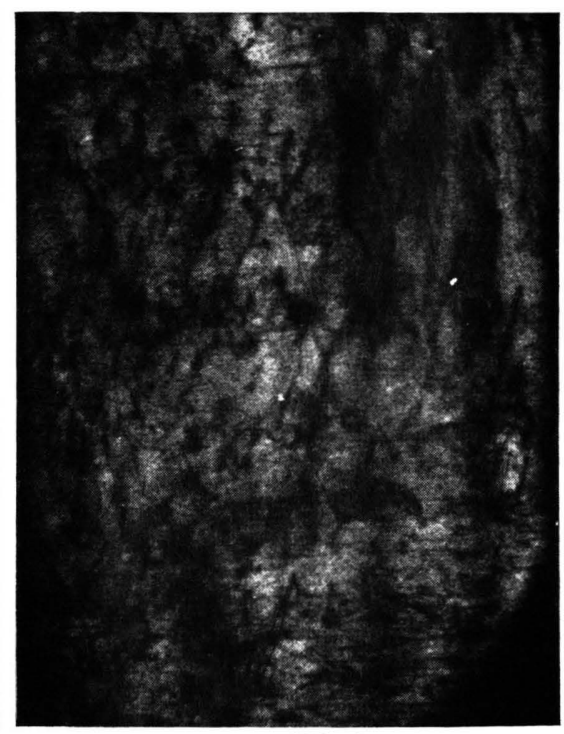

Abb. 4.
Abb. 2. Parallelstrahlaufnahme an einem idealen Quarzkristall mit einer Drahtgitterblende im primären Strahl (Stellung $G_{1}$ ), 10,5-fach vergrößert, polierte Oberflächen 2354, Richtung anomaler Absorption 1101, Cu K-Strahlung, $25 \mathrm{kV}$, $10 \mathrm{~mA}, 4 \mathrm{~h}$. Der Strichfokus liegt parallel zur horizontalen Linie. Die drei Pfeile zeigen auf Emulsionsfehler. Alles andere sind Oberflächenstörungen.
Abb. 3. Parallelstrahlaufnahme an einem idealen Quarzkristall wie in Abb. 2 jedoch mit einer Drahtgitterblende im austretenden Strahl R (Stellung $\mathrm{G}_{2}$ ). Vergrößerung 10,5.

Abb. 4. Parallelstrahlaufnahme nach Abb. 1 an einem $\mathrm{Si}$ Kristall mit 2500 Ätzgruben pro $\mathrm{cm}^{2}$, 10,5-fach vergrößert, $1 \mathrm{~mm}$ stark, blankgeätzte 111-Oberfläche, Richtung anomaler Absorption 220, Cu K-Strahlung, $25 \mathrm{kV}, 10 \mathrm{~mA}, 8 \mathrm{~h}$ **. 
von Abdrücken für höchste Auflösung durchzuführen wären.

In der Abb. 3 wird eine Aufnahme von erstmals aufgefundenen Spaltstrukturen mitgeteilt, die mit der hier mitgeteilten Methode häufiger zu beobachten sind. Auch hier sind diese - an nicht vorbehandelten Spaltflächen durch übliche Abdruckherstellung nicht zu erfassenden - Spaltstrukturen durch ein bevorzugtes Wachstum an den Stufen zur Abbildung gebracht. Die auf dieser Aufnahme zu beobachtenden Spaltstrukturen sind durch eine Wechselwirkung der schräg von oben anlaufenden sehr feinen Spaltstufen mit einer kreuzenden Spaltstufe entstanden. Die entgegengesetzte Auslenkung der feinen Spaltstufen ober- und unterhalb der durchquerten Spaltstufe sollte durch in der letzteren angeordnete und während der Spaltung betätigte Versetzungen bewirkt sein. Ähnliche Strukturen hat GRIFriN ${ }^{10}$ - allerdings in größeren Abmessungen - lichtmikroskopisch an Beryll-Kristallen aufgefunden. Griffin gibt eine Erklärung, die auf eine Wechselwirkung von Frank-ReadQuellen während des Wachstumsvorganges mit Wachs- tumslamellen basiert. In Abb. 3 sind die schräg nach unten verlaufenden Stufen mit Sicherheit keine Kanten von Wachstumslamellen; aber es ist sehr wohl möglich, daß die in der Griffinschen Vorstellung notwendige, durch den Wachstumsvorgang gelieferte Bewegungsmöglichkeit der Gitterbausteine, also auch der Stufen, in unserem Fall während der Bruchausbreitung bei der Kristallspaltung, gegeben ist. Diese Strukturen sind damit von besonderem Interesse für das Studium des Spaltvorganges und des Sprödbruches. Eine ausführliche Diskussion hierzu ist in Vorbereitung.

Die hier beschriebene Methode zur Sichtbarmachung von Versetzungen und üblicherweise mit der Abdrucktechnik nicht zu erfassenden Oberflächenstrukturen sollte allgemein auf spaltbare Kristalle anzuwenden sein. Eine Untersuchung an Germanium wird zur Zeit durchgeführt.

Herrn Prof. Dr. Messerschmidt danken wir sehr für das dieser Arbeit entgegengebrachte Interesse.

10 L. I. Griffin, Phil. Mag. 43, 827 [1952].

\section{Zum Wachstum von Kupferoxydnadeln}

\author{
Von W. D. Dachselt und G. Pfefferkorn \\ Abteilung für Medizinische Elektronenmikroskopie \\ an der Universität Münster (Westf.) \\ (Z. Naturforschg. 14 a, 309 [1959]; eingegangen am 14. März 1959)
}

In Fortsetzung der Forschungen über das Wachstum von sublichtmikroskopischen Oxydnadeln betrachteten die Verfasser zunächst mit Hilfe von Kohlehüllen und Dünnschnitten elektronenmikroskopisch den Habitus von Kupferoxydnadeln. Diese wurden allseitig mit Kohle bedampft und anschließend das Oxyd herausgelöst. Stereoskopische Aufnahmen der Kohlehüllen zeigten eine polygonale, oft sechsseitige Begrenzung der Nadeln. Weiterhin wurden die in polymerisiertem Metacrylat eingebetteten Nadeln senkrecht zur Nadelachse mit dem Diamantmesser des Leitz-Ultramikrotoms geschnitten. Auch hier waren die Nadeln meist sechsseitig begrenzt. Hohle Nadeln konnten bisher nicht gefunden werden.

Die kristallographische Richtung der Nadelachse sollte durch Feinstrukturuntersuchung mit RönTGEN- und Elektronen-Strahlen bestimmt werden. Zur Oxydnadelherstellung wurden zwei Präparationsmethoden verwen. det.

1. Das Kupfer wurde, wie bisher üblich, im Luft- bzw. Sauerstoffstrom oxydiert. Durchschnittlich hatten die Nadeln einen Durchmesser von $0,1 \mu$ und konnten daher nur mit Elektronenbeugung untersucht werden.

2. In Anwesenheit von HCl-Dämpfen erhielt man an der Kontaktstelle von Kupfer mit Silber bei niedrigeren Temperaturen als bei der reinen Sauerstoffoxydation wesentlich dickere, mikroskopisch sichtbare $\mathrm{CuO}-\mathrm{Nadeln}$. Diese dicken Nadeln eigneten sich auch zur röntgenographischen Untersuchung.
Mit Elektronenbeugung wurden sowohl die in Luft bzw. im Sauerstoffstrom gebildeten Nadeln als auch die dickeren unter $\mathrm{HCl}-Z$ usatz gewonnenen Oxydnadeln untersucht. Die fast senkrecht aus der oxydierten Kupferoberfläche herauswachsenden Nadeln zeigten bei der Elektronenbeugung auf Grund ihrer angenähert parallelen Orientierung ein Texturdiagramm mit Schichtlinien (Abb. $1^{*}$ ). Abb. 2 zeigt das Beugungsdiagramm vorwiegend von einer Nadel.

Die Vermessung des Schichtlinienabstandes ergab an den in Luft bzw. Sauerstoff entstandenen Oxydnadeln und an den unter HCl-Einwirkung gewachsenen Nadeln die gleiche Translationsperiode in Nadelrichtung $T=2,87 \pm 0,02 \AA$ A. Damit ist sichergestellt, daß die $\mathrm{Zu}-$ gabe von $\mathrm{HCl}$ das Wachstum wesentlich begünstigt, ohne die Wachstumsrichtung zu ändern. Der gefundene Wert von $2,87 \AA$ entspricht am besten der errechneten Translationsperiode von $2,88 \AA$ in Richtung [110] des monoklinen Kupferoxydgitters. Zur sicheren Feststellung der Wachstumsrichtung wurde eine RöNTGEN-Drehkristallaufnahme um die Achse einer mikroskopisch einjustierten Nadel von $20 \mu$ Durchmesser gemacht $\left(\mathrm{CuK}_{a}\right.$, $40 \mathrm{kV}, 20 \mathrm{~mA}$, Belichtungszeit 3 Tage). Aus dem Schichtlinienabstand ergab sich eine Translationsperiode von $2,87 \pm 0,02 \AA$, die mit den Werten der Elektronenbeugungsdiagramme übereinstimmt. Entsprechend der Strukturbestimmung von Kupferoxyd durch Tunell, Posnjak und Ksanda ${ }^{1}$ wurde die nullte und erste Schichtlinie der Drehkristallaufnahme indiziert. Daraus ergab sich ebenfalls die Wachstumsrichtung [110]. Eine weitere Aufklärung der Wachstumsgesetze der Kupferoxydnadeln ist im Gange.

1 G. Tunell, E. Posnjak u. C. J. Ksanda, Z. Kristallographie 90, 121 [1935].

* Abb. 1 u. 2 auf Tafel S. 308 b. 